\title{
Little consensus on egg freezing
}

\section{WASHINGTON DC}

Freezing a woman's eggs for use later in fertility treatments doesn't seem to greatly increase birth defects or abnormalities, new data suggest. But the findings, presented at the annual meeting of the American Society for Reproductive Medicine (ASRM) in Washington DC last week, are difficult to interpret. The field remains caught between researchers, most of whom say that the practice should still be considered experimental, and the public, which is itching to embrace the technology to extend a woman's fertile years.

Egg freezing is regarded as a positive, if costly, option for women facing illness or treatment that could truncate their fertility. But most researchers think that the procedure is not appropriate for women who wish to delay fertility for social, career or other non-health-related reasons.

"We acknowledge the interest women have in this technology," says Marc Fritz, a professor of obstetrics and gynaecology at the University of North Carolina at Chapel Hill. But "there remain very few studies in which the safety and efficacy of this practice can be judged."

Last week, an ASRM committee chaired by Fritz recommended that egg freezing continue to be considered experimental, and not be marketed or promoted to healthy young women.

Human eggs are large, fragile and filled with water, and both freezing and thawing can damage them. Freezing fertilized embryos is easier and more routine, but is less desirable for some women owing to social or ethical reasons. Some women wish to preserve eggs for the right partner, and couples can be uncomfortable with the prospect of freezing embryos.

Egg freezing, which has been

\section{"Every scrap of} evidence comes with a dizzying number of caveats." in place for at least two decades, has not been used widely; doctors estimate that between 300 and 600 children have been born from formerly frozen eggs.

Safety remains one of the main concerns. At the ASRM meeting, Andrea Borini of the Centre for Reproductive Health in Bologna, Italy, reported that just 2 of 123 live births from his clinic's egg cryopreservation programme had major abnormalities: one had a nasal blockage, the other a rare developmental disorder called Rubinstein-Taybi Syndrome. (But both children had fathers with fertility problems; "we may need to look into that," says Borini.)

Meanwhile, Ilan Tur-Kaspa of the Reproductive Genetics Institute in Chicago, Illinois, pre-

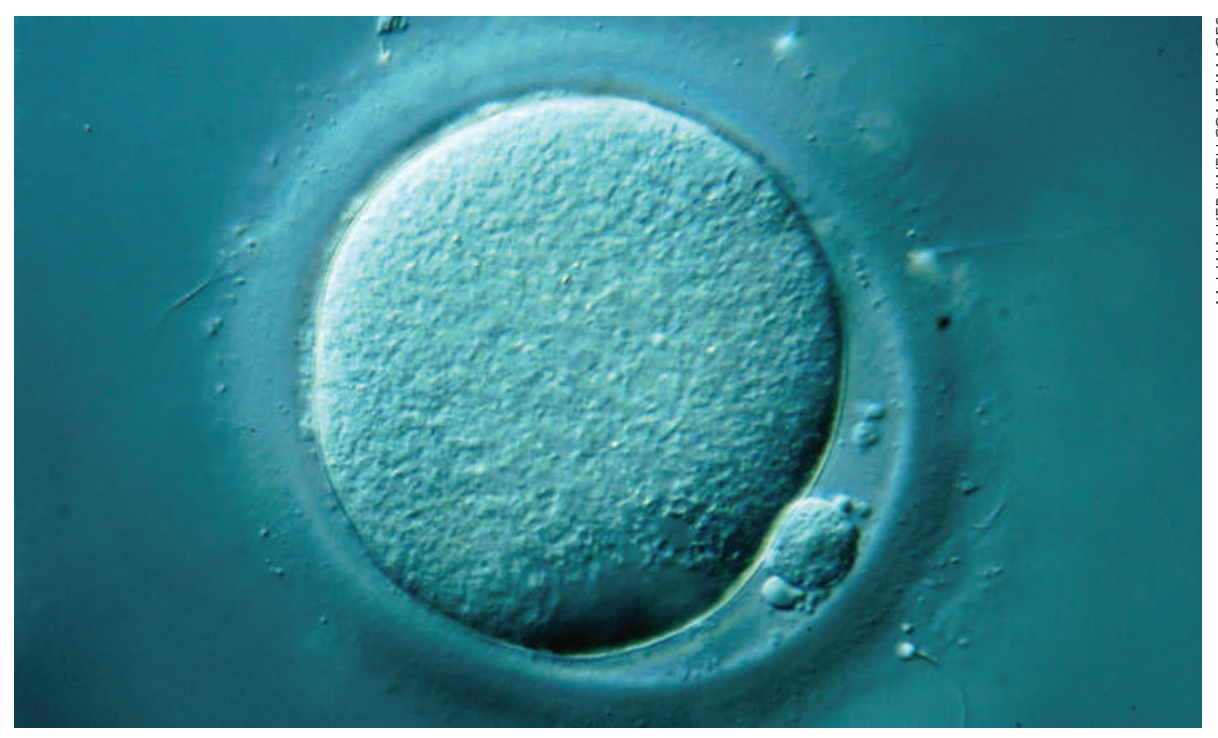

Experts disagree on the safety and efficacy of egg-freezing techniques.

sented an analysis of more than 37 publications on children born from cryopreserved eggs. Of 555 live births, including those reported by Borini's group, he turned up just 3 other major abnormalities.

Demonstrating just how difficult the data are to interpret, Kutluk Oktay from Weill Cornell Medical College in New York says that his group tallies the number of reported births from cryopreserved eggs at closer to 300 , not 555 . He says that the Tur-Kaspa group missed some overlap in the studies - places where researchers re-reported earlier findings or where collaborative groups reported on each others' data. But Tur-Kaspa says that they looked closely for these instances, and says that "as far as we know there's no overlap".

Nevertheless, the data are reassuring, TurKaspa says. Five abnormalities in 550 births is a less than $1 \%$ abnormality rate - comparable to that for a natural pregnancy. The study didn't address efficacy, though. "We don't know the denominator - we don't know how many [pregnancies] were terminated," says Oktay. "It gives you a summary of how many babies are out there and how many might have an abnormality. But none of the data we've seen out there is sufficient to say the issue is resolved."

One problem in comparing studies is that eggs can be frozen several ways. The method Borini's group uses, known as slow-rate freezing, automatically cools the eggs in stages to subzero tem- peratures, in the hope that water will be forced out of the egg before it crystallizes. Another process, vitrification, is a flash-freezing process that requires more manual involvement.

\section{Cool tactics}

Many practitioners think that vitrification is the superior technology, but few prospective studies have been done to compare the procedures. To help change that, Gary Smith, director of the Assisted Reproductive Technologies Laboratory at the University of Michigan in Ann Arbor, has been collaborating with a group at the Huntington Centre for Reproductive Medicine in São Paulo, Brazil. At the ASRM meeting, Smith presented results from a small study in which 28 slow-frozen eggs, and 35 vitrified ones, were thawed then fertilized. Further analysis of the results indicates that for every 21 oocytes frozen via vitrification, one pregnancy could be expected. The numbers were slightly worse for slow-rate freezing: 45 frozen eggs per pregnancy.

Doubts remain about egg cryopreservation, and every scrap of evidence comes with a dizzying number of caveats because of variability both in procedures and in patients. Still, some doctors are ready to broaden its use. "We're kind of at a point in this technology where it's kind of like a teeter totter," says Smith. "For me the important thing is the completion of studies like this that give the patients information about the reality of these technologies."

Brendan Maher 